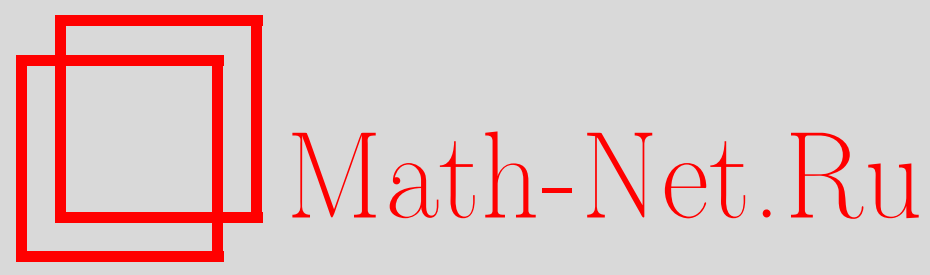

Ю. Ю. Бахтин, Закон повторного логарифма для решения уравнения Бюргерса со случайными начальными данными, Матем. заметки, 1998, том 64, выпуск 6, 812-823

DOI: https://doi.org/10.4213/mzm1460

Использование Общероссийского математического портала Math-Net.Ru подразумевает, что вы прочитали и согласны с пользовательским соглашением http://www.mathnet.ru/rus/agreement

Параметры загрузки:

IP : 54.198 .55 .26

26 апреля 2023 г., 18:37:29 


\title{
ЗАКОН ПОВТОРНОГО ЛОГАРИФМА ДЛЯ РЕШЕНИЯ УРАВНЕНИЯ БЮРГЕРСА СО СЛУЧАЙНЫМИ НАЧАЛЬНЫМИ ДАННЫМИ
}

\author{
Ю. Ю. Бахтин
}

Для решения одномерного уравнения Бюргерса в случае, когда начальный потенциал описьвается дробовьм шумом нулевого радиуса взаимодействия, устанавливается закон повторного логарифма.

Библиографоия: 14 названий.

При изучении поведения нелинейных волн в средах без дисперсии эталонным является многомерное уравнение Бюргерса. А именно, изучается задача Коши

$$
\begin{gathered}
\frac{\partial v}{\partial t}+(v, \nabla) v=\kappa \Delta v, \\
v(0, x)=-2 \kappa \nabla \xi(x), \quad(t, x) \in \mathbb{R}_{+} \times \mathbb{R}^{d}, \quad v(t, x) \in \mathbb{R}^{d},
\end{gathered}
$$

где $\kappa>0$ - параметр вязкости. Это уравнение и его аналоги описьвают волновые явления различной физической природы: нелинейные акустические и оптические волны, гидродинамические потоки частиц, турбулентные явления. Оно играет центральную роль в гидродинамическом подходе к изучению неоднородного распределения материи во Вселенной. При этом по физическим соображениям наибольший интерес представляет потенциальньй случай, когда $v(t, x)=\nabla \Phi(t, x)$, где $\nabla$-градиент по $x$ (см., например, монографию [1] и указанную в ней библиографию).

В последние годы многочисленные исследования посвящены асимптотическому поведению решений или преобразованных решений уравнения Бюргерса со случайными начальньпи данными различных типов (см. [2]-[8] и ссылки в этих работах).

Нас будет интересовать характер флуктуаций решений одномерного $(d=1)$ уравнения Бюргерса в случае, когда начальные данные описьваются процессом дробового шума. Это направление инициировано работой [2] и получило дальнейшее развитие в [3], [4], где установлена центральная предельная теорема для преобразованных решений задачи (1). Основное содержание этой работы - доказательство закона повторного логарифма, т.е. определение точных границ, описывающих с вероятностью 1 характер флуктуаций исследуемого случайного процесса, когда начальные данные задаются дробовым шумом с нулевым радиусом взаимодействия.

Нам понадобятся некоторые определения и вспомогательные утверж дения. 
Пусть $\mathscr{B}$ - борелевская $\sigma$-алгебра на прямой, а $\mathscr{B}_{b}$ - семейство ограниченных борелевских множеств. На множестве $M$ всех мер $\mu$, определенных на $(\mathbb{R}, \mathscr{B})$ и конечных на ограниченных множествах, определим $\sigma$-алгебру $\mathscr{M}$, порожденную множествами $\{\mu \in M: \mu(A) \leqslant r\}$ для $A \in \mathscr{B}_{b}, r \in \mathbb{R}_{+}=[0, \infty)$. Случайная мера $\mu$ - измеримое отображение из вероятностного пространства $(\Omega, \mathscr{F}, \mathrm{P})$ в $(M, \mathscr{M})$. Таким образом, для любого $A \in \mathscr{B}_{b} \quad \mu(A)$ - неотрицательная случайная величина в обычном смысле.

Пусть $\lambda>0$ и случайная мера $\mu$ удовлетворяет следующим двум условиям:

1) для любых непересекающихся множеств $A_{1}, \ldots, A_{m} \in \mathscr{B}_{b}$ случайные величины $\mu\left(A_{1}\right), \ldots, \mu\left(A_{m}\right)$ независимы;

2) для любого множества $A \in \mathscr{B}_{b}$ величина $\mu(A)$ распределена так же, как $\sum_{i=1}^{\pi(A)} \eta_{i}$, где $\pi(A)$ - пуассоновская случайная величина с параметром $\lambda|A|(|A|$ - мера Лебега множества $A)$, а $\left(\eta_{i}\right)_{i=1}^{\infty}-$ независимые неотрицательные случайные величины с одинаковой функцией распределения $F$, независимые от $\pi(A)$ (здесь и далее сумму, взятую по пустому множеству, считаем равной 0 ).

Тогда такую меру $\mu$ будем назьвать дробовым шумом с нулевым радиусом взаимодействия или вырожденным дробовым шумом. Ее можно построить по возрастающему и непрерывному справа процессу $(Z(x))_{x \in \mathbb{R}}$ с независимыми приращениями, для которого $\operatorname{Exp}(i r(Z(x)-Z(y)))=\exp \left(\lambda(x-y)\left(\varphi_{\eta}(r)-1\right)\right)$, где $\varphi_{\eta}(r)=\mathrm{E} \exp \left(i r \eta_{1}\right)$.

Если $\eta_{i}=1$ почти наверное, то $\mu$ - стандартная пуассоновская мера с ведущей мерой Лебега и параметром $\lambda$.

Общие процессы дробового шума рассматриваются, например, в [9].

ПРЕДЛОЖЕНИЕ 1. Пусть $\mu$ - дробовой шум с нулевым радиусом взаимодействия. Обозначим через $\mathscr{F}_{a}^{b} \sigma$-подалгебру, порожденную случайными величинами $\mu\left(a^{\prime}, b^{\prime}\right],\left(a^{\prime}, b^{\prime}\right] \subset(a, b]$. Тогда для непересекаюшихся отрезков $\left(a_{1}, b_{1}\right] u\left(a_{2}, b_{2}\right]$ подалгебры $\mathscr{F}_{a_{1}}^{b_{1}}$ и $\mathscr{F}_{a_{2}}^{b_{2}}$ независимы как порожденные независимыми случайными величинами. В частности, интегралы по непересекаюшимся отрезкам от неслучайных измеримых функиий по вырожденному дробовому иуму независимы, если эти интегралы существуют почти наверное.

Теперь вернемся к уравнению Бюргерса. Не ограничивая общности, положим для простоты записи $\kappa=1 / 2$. Тогда задача (1) в случае $d=1$ допускает решение

$$
v(t, x)=\int_{\mathbb{R}} \frac{x-y}{t} \exp \left(\xi(y)-\frac{(x-y)^{2}}{2 t}\right) d y / \int_{\mathbb{R}} \exp \left(\xi(y)-\frac{(x-y)^{2}}{2 t}\right) d y
$$

которое можно получить с помощю подстановки Хопфа-Коула

$$
v=-\frac{\partial}{\partial x} \ln u
$$

Формальная запись $e^{\xi(x)} d y=\mu(d y)$ означает, что формула (2) принимает вид

$$
v(t, x)=\int_{\mathbb{R}} \frac{x-y}{t} \exp \left(-\frac{(x-y)^{2}}{2 t}\right) \mu(d y) / \int_{\mathbb{R}} \exp \left(-\frac{(x-y)^{2}}{2 t}\right) \mu(d y),
$$

где $\mu$ - случайная мера, описьвающая дробовой шум с нулевым радиусом взаимодействия. 
Пусть распределение величин $\eta_{i}$ сосредоточено на некотором отрезке $[0, M], M>0$. Интегралы в числителе и знаменателе сходятся почти наверное. Действительно, для $\delta>0$

$$
\begin{aligned}
\int_{\mathbb{R}} \frac{x-y}{t} \exp \left(-\frac{(x-y)^{2}}{2 t}\right) \mu(d y) & =\sum_{k \in \mathbb{Z}} \int_{(k \delta,(k+1) \delta]} \frac{x-y}{t} \exp \left(-\frac{(x-y)^{2}}{2 t}\right) \mu(d y) \\
& =\sum_{k \in \mathbb{Z}} X_{k, \delta}(t)
\end{aligned}
$$

- сумма независимых (в силу предложения 1) случайных величин. Вычисление дисперсий, проводимое ниже при доказательстве основного результата, даст

$$
\sum_{k \in \mathbb{Z}} \mathrm{D} X_{k, \delta}(t)<\infty
$$

Так как $\sum_{k \in \mathbb{Z}} \mathrm{E} X_{k}(t)=0$, по теореме о двух рядах (см., например, [10, с. 373]) ряд сходится почти наверное. Аналогичные рассуждения применимы и для знаменателя (3). Теперь можно сформулировать основной результат.

ТЕорема. Пусть решение $v(t, x)$ определено формулой (3) и распределение случайных величин $\eta_{i}$ сосредоточено на некотором отрезке $[0, M], M>0$. Тогда для любого $x \in \mathbb{R}$

$$
\varlimsup_{t \rightarrow \infty} \frac{|v(t, x)|}{C t^{-3 / 4}(\ln \ln t)^{1 / 2}}=1 \quad \text { почти наверное, } \quad \text { дее } \quad C=\frac{\left(\mathrm{E} \eta^{2}\right)^{1 / 2}}{(2 \lambda)^{1 / 2} \pi^{1 / 4} \mathrm{E} \eta}
$$

( $\eta$ - случайная величина с функиией распределения $F)$.

ДоКАЗАТЕЛЬСТВо состоит в установлении закона повторного логарифма для числителя (3) и усиленного закона больших чисел для знаменателя (3).

ЗАмЕчАниЕ. В [2] при доказательстве центральной предельной теоремы для уравнения Бюргерса также отдельно рассматривались числитель и знаменатель (2). Для числителя устанавливалась асимптотическая гауссовость, а для знаменателя - закон больших чисел. Здесь нам требуется именно усиленная версия закона больших чисел.

Сначала рассмотрим числитель (3).

Пусть $x=0$, что не ограничивает общности рассуждений. Итак, положим для $\Delta>0$

$$
V(t)=\int_{\mathbb{R}} x \exp \left(-\frac{x^{2}}{2 t}\right) \mu(d x)=\sum_{k \in \mathbb{Z}} \int_{(k \Delta,(k+1) \Delta]} x \exp \left(-\frac{x^{2}}{2 t}\right) \mu(d x)=\sum_{k \in \mathbb{Z}} X_{k, \Delta}(t) .
$$

Отметим, что семиинварианты $V(t)$ распадаются в сумму соответствующих семиинвариантов $X_{k, \Delta}(t)$, так как в силу предложения $1 X_{k, \Delta}(t)$ независимы при различных $k$.

Для вычисления семиинвариантов $\gamma_{\nu}(V(t)), \nu \in \mathbb{N}$, оценим сначала моменты $X_{k, \Delta}(t)$. Пусть

$$
\begin{array}{ll}
\bar{X}_{k, \Delta}(t)=a_{k, \Delta}^{-}(t) \mu(k \Delta,(k+1) \Delta], \quad \text { где } a_{k, \Delta}^{-}(t)=\inf _{x \in(k \Delta,(k+1) \Delta]} x \exp \left(-\frac{x^{2}}{2 t}\right), \\
\widehat{X}_{k, \Delta}(t)=a_{k, \Delta}^{+}(t) \mu(k \Delta,(k+1) \Delta], \quad \text { где } a_{k, \Delta}^{+}(t)=\sup _{x \in(k \Delta,(k+1) \Delta]} x \exp \left(-\frac{x^{2}}{2 t}\right) .
\end{array}
$$


Тогда, полагая $A_{0}=\sup _{x \in \mathbb{R}} x \exp \left(-x^{2} /(2 t)\right)$,

$$
\begin{aligned}
\left|\mathrm{E} \bar{X}_{k, \Delta}^{\nu}(t)-\mathrm{E} X_{k, \Delta}^{\nu}(t)\right| & \leqslant \mathrm{E}\left|\bar{X}_{k, \Delta}^{\nu}(t)-\widehat{X}_{k, \Delta}^{\nu}(t)\right| \\
& \leqslant\left|a_{k, \Delta}^{+}(t)^{\nu}-a_{k, \Delta}^{-}(t)^{\nu}\right| \mathrm{E} \mu(k \Delta,(k+1) \Delta]^{\nu} \\
& \leqslant\left|a_{k, \Delta}^{+}(t)-a_{k, \Delta}^{-}(t)\right| \sup _{|y| \leqslant A_{0}}\left|\nu y^{\nu-1}\right| \sum_{m=1}^{\infty} e^{-\lambda \Delta} \frac{(\lambda \Delta)^{m}}{m !}(m M)^{\nu} \\
& \leqslant\left|a_{k, \Delta}^{+}(t)-a_{k, \Delta}^{-}(t)\right| A_{1}(\nu, \lambda, M, t) \Delta
\end{aligned}
$$

при малых $\Delta\left(\Delta \leqslant \Delta_{0}(\nu)\right)$.

Из формулы (см. [11, с. 20])

$$
\gamma_{\nu}(\xi)=\nu ! \sum(-1)^{r_{\nu}-1}\left(r_{\nu}-1\right) ! \prod_{l=1}^{\nu} \frac{1}{m_{l} !}\left(\frac{\alpha_{l}(\xi)}{l !}\right)^{m_{l}}
$$

связьвающей моменты $\alpha_{l}$ и семиинварианты $\gamma_{\nu}$ случайной величины $\xi\left(r_{\nu}=m_{1}+m_{2}+\right.$ $\cdots+m_{\nu}$, а суммирование производится по целым неотрицательным решениям уравнения $\left.m_{1}+2 m_{2}+\cdots+\nu m_{\nu}=\nu\right)$, и из (4) следует, что для малых $\Delta\left(\Delta \leqslant \Delta_{1}(\nu)\right)$

$$
\left|\gamma_{\nu}\left(\bar{X}_{k, \Delta}(t)\right)-\gamma_{\nu}\left(X_{k, \Delta}(t)\right)\right| \leqslant A_{2}(\nu, \lambda, M, t)\left|a_{k, \Delta}^{+}(t)-a_{k, \Delta}^{-}(t)\right| \Delta,
$$

так как в многочлене от $\alpha_{1}, \ldots, \alpha_{\nu}$, стоящем в правой части (5), только одно слагаемое имеет первую степень: $\alpha_{\nu}$. Остальные члены имеют степень не меньше 2 и в сумме дают величину порядка $O\left(\Delta^{2}\right)$. Пусть $h \delta=\Delta$. Тогда

$$
\begin{aligned}
\left|\gamma_{\nu}\left(X_{k, \Delta}(t)\right)-\sum_{l=k h}^{(k+1) h-1} \gamma_{\nu}\left(\bar{X}_{l, \delta}(t)\right)\right| & \leqslant A_{2}(\nu, \lambda, M, t) \sum_{l=k h}^{(k+1) h-1}\left|a_{l, \delta}^{+}(t)-a_{l, \delta}^{-}(t)\right| \delta \\
& \leqslant A_{2}(\nu, \lambda, M, t) D(t) \delta,
\end{aligned}
$$

где $D(t)$ - полная вариация функции $x \exp \left(-x^{2} /(2 t)\right)$ на прямой $(D(t)<\infty)$.

Далее,

$$
\begin{aligned}
\mathrm{E} \bar{X}_{l, \delta}^{\nu}(t) & =\sum_{m=0}^{\infty} e^{-\lambda \delta} \frac{(\lambda \delta)^{m}}{m !} a_{l, \delta}^{-}(t)^{\nu} \mathrm{E}\left(\left(\sum_{i=1}^{\pi(A)} \eta_{i}\right)^{\nu} \mid \pi(A)=m\right) \\
& \leqslant \sum_{m=1}^{\infty} e^{-\lambda \delta} \frac{(\lambda \delta)^{m}}{m !}\left(m M\left|a_{l, \delta}^{-}(t)\right|\right)^{\nu} \leqslant A_{3}(\nu, \lambda, M, t) \delta
\end{aligned}
$$

для малых $\delta\left(\delta \leqslant \delta_{0}(\nu)\right)$.

Поэтому из формулы (5) следует, что

$$
\left|\gamma_{\nu}\left(\bar{X}_{l, \delta}(t)\right)-\mathrm{E} \bar{X}_{l, \delta}(t)^{\nu}\right| \leqslant A_{4}(\nu, \lambda, M, t) \delta^{2} .
$$


В свою очередь,

$$
\begin{aligned}
\mathrm{E} \bar{X}_{l, \delta}^{\nu}(t) & =\sum_{m=0}^{\infty} e^{-\lambda \delta} \frac{(\lambda \delta)^{m}}{m !} a_{l, \delta}^{-}(t)^{\nu} \mathrm{E}\left(\left(\sum_{i=1}^{\pi(A)} \eta_{i}\right)^{\nu} \mid \pi(A)=m\right) \\
& =e^{-\lambda \delta} \lambda \delta \mathrm{E} \bar{\eta}_{1}^{\nu} a_{l, \delta}^{-}(t)^{\nu}+\sum_{m=2}^{\infty} e^{-\lambda \delta} \frac{(\lambda \delta)^{m}}{m !} a_{l, \delta}^{-}(t)^{\nu} \mathrm{E}\left(\left(\sum_{i=1}^{\pi(A)} \bar{\eta}_{i}\right)^{\nu} \mid \pi(A)=m\right) .
\end{aligned}
$$

В равенстве (8) сумма $\sum_{m=2}^{\infty}$ не превосходит по модулю

$$
S_{\delta}=\sum_{m=2}^{\infty} e^{-\lambda \delta} \frac{(\lambda \delta)^{m}}{m !}\left(m M\left|a_{l, \delta}^{-}(t)\right|\right)^{\nu} \leqslant A_{5}(\nu, \lambda, M, t) \delta^{2}
$$

при малых $\delta\left(\delta \leqslant \delta_{1}(\nu)\right)$. Теперь из (6)-(9) следует, что

$$
\left|\gamma_{\nu}\left(X_{k, \Delta}(t)\right)-\lambda \mathrm{E} \bar{\eta}_{1}^{\nu} \sum_{l=k h}^{(k+1) h-1} a_{l, \delta}^{-}(t)^{\nu} \delta\right| \leqslant A_{6}(\nu, \lambda, M, t, \Delta) \delta
$$

при малых $\delta, \delta \leqslant \min \left(\delta_{0}(\nu), \delta_{1}(\nu)\right)$. Поэтому

$$
\lambda \mathrm{E} \bar{\eta}_{1}^{\nu} \sum_{l=k h}^{(k+1) h-1} a_{l, \delta}^{-}(t)^{\nu} \delta \rightarrow \gamma_{\nu}\left(X_{k, \Delta}(t)\right) \quad \text { при } \delta \rightarrow 0
$$

Однако,

$$
\sum_{l=k h}^{(k+1) h-1} a_{l, \delta}^{-}(t)^{\nu} \delta
$$

является интегральной суммой для непрерьвной функции $x^{\nu} \exp \left(-x^{2} \nu /(2 t)\right)$. Поэтому

$$
\gamma_{\nu}\left(X_{k, \Delta}(t)\right)=\lambda \mathrm{E} \bar{\eta}_{1}^{\nu} \int_{k \Delta}^{(k+1) \Delta} x^{\nu} \exp \left(-\frac{x^{2} \nu}{2 t}\right) d x
$$

Значит,

$$
\begin{aligned}
\gamma_{\nu}(V(t)) & =\lambda \mathrm{E} \bar{\eta}_{1}^{\nu} \int_{\mathbb{R}} x^{\nu} \exp \left(-\frac{x^{2} \nu}{2 t}\right) d x \\
& =\left\{\begin{array}{ll}
\lambda \mathrm{E} \bar{\eta}_{1}^{2 n} \sqrt{2 \pi \frac{t}{2 n}}(2 n-1) ! !\left(\frac{t}{2 n}\right)^{n}, & \text { если } \nu=2 n, \\
0, & \text { если } \nu=2 n-1,
\end{array} \quad n \in \mathbb{N},\right.
\end{aligned}
$$


Далее,

$$
\begin{aligned}
\mathrm{E} e^{i r V(t) / t^{3 / 4}} & =\exp \left(\sum_{n=1}^{\infty}(-1)^{n} \lambda \mathrm{E} \bar{\eta}_{1}^{2 n} \sqrt{2 \pi} \frac{t^{(1-n) / 2} r^{2 n}}{(2 n)^{n+1 / 2}(2 n) ! !}\right) \\
& =\exp \left(-\lambda \mathrm{E} \bar{\eta}_{1}^{2} \sqrt{2 \pi} \frac{r^{2}}{2^{3 / 2} 2}\right) \exp \left(\sum_{n=2}^{\infty}(-1)^{n} \lambda \mathrm{E} \bar{\eta}_{1}^{2 n} \sqrt{2 \pi} \frac{t^{(1-n) / 2} r^{2 n}}{(2 n)^{n+1 / 2}(2 n) ! !}\right) .
\end{aligned}
$$

При $|r M| \leqslant t^{1 / 8}$

$$
\begin{aligned}
& \left|\sum_{n=2}^{\infty}(-1)^{n} \lambda \mathrm{E} \bar{\eta}_{1}^{2 n} \sqrt{2 \pi} \frac{t^{(1-n) / 2} r^{2 n}}{(2 n)^{n+1 / 2}(2 n) ! !}\right| \leqslant r^{4} M^{4} \lambda \sqrt{2 \pi} \sum_{n=2}^{\infty} \frac{t^{(1-n) / 2}|r M|^{2 n-4}}{(2 n)^{n+1 / 2}(2 n) ! !} \\
& \quad=r^{4} M^{4} \lambda \sqrt{2 \pi} \sum_{n=2}^{\infty} \frac{t^{(1-n) / 2} t^{n / 4-1 / 2}}{(2 n)^{n+1 / 2}(2 n) ! !}=r^{4} M^{4} \lambda \sqrt{2 \pi} \sum_{n=2}^{\infty} \frac{t^{-n / 4}}{(2 n)^{n+1 / 2}(2 n) ! !} \\
& \quad=r^{4} M^{4} \lambda \sqrt{2 \pi} t^{-1 / 2} \sum_{n=2}^{\infty} \frac{t^{1 / 2-n / 4}}{(2 n)^{n+1 / 2}(2 n) ! !} .
\end{aligned}
$$

Так как в правой части формулы (11) сумма представляет собой степенной ряд от $t^{-1 / 4}$ с бесконечным радиусом сходимости, при $t>1$ она не превосходит некоторой константы $A_{7}$. Итак, при $|r M| \leqslant t^{1 / 8}$ и $t>1$

$$
\left|\sum_{n=2}^{\infty}(-1)^{n} \lambda \mathrm{E} \bar{\eta}_{1}^{2 n} \sqrt{2 \pi} \frac{t^{(1-n) / 2} r^{2 n}}{(2 n)^{n+1 / 2}(2 n) ! !}\right| \leqslant A_{8} t^{-1 / 2}(r M)^{4} .
$$

Из (10) и (12) следует, что

$$
\begin{aligned}
& \left|\mathrm{E} e^{i r V(t) / t^{3 / 4}}-\exp \left(-\lambda \mathrm{E} \bar{\eta}_{1}^{2} \sqrt{2 \pi} \frac{r^{2}}{2^{3 / 2} 2}\right)\right| \\
& \quad \leqslant \exp \left(-\lambda \mathrm{E} \bar{\eta}_{1}^{2} \sqrt{2 \pi} \frac{r^{2}}{2^{3 / 2} 2}\right)\left(\left|1-e^{A_{8} t^{-1 / 2}(r M)^{4}}\right| \mathrm{V}\left|1-e^{-A_{8} t^{-1 / 2}(r M)^{4}}\right|\right) \\
& \quad \leqslant A_{9} t^{-1 / 2}(r M)^{4} \exp \left(-\lambda \mathrm{E} \bar{\eta}_{1}^{2} \sqrt{2 \pi} \frac{r^{2}}{2^{3 / 2} 2}\right)
\end{aligned}
$$

для $|r M| \leqslant t^{1 / 8}$ и $t>1$.

Воспользуемся теперь следующим утверждением (см. [11, с. 154]).

ПредЛоЖениЕ 2. Пусть $F(x)$ - неубъвающая ограниченная функиия, $G(x)$ дифференцируемая функиия ограниченной вариации на $\mathbb{R}, f(t)$ и $g(t)$ - соответствующие им преобразования Фурье-Стилтьеса, $F(-\infty)=G(-\infty)=0, T-n р о-$ извольное положительное число. Пусть $\sup _{x}\left|G^{\prime}(x)\right| \leqslant C$. Тогда для любого числа $b>1 /(2 \pi)$ имеет место неравенство

$$
\sup |F(x)-G(x)| \leqslant b \int_{-T}^{T}\left|\frac{f(t)-g(t)}{t}\right| d t+r(b) \frac{C}{T},
$$


әде $r(b)$ - положительная постоянная, зависящая только от $b$.

Пусть $F(x)=\mathrm{P}\left\{V(t) / t^{3 / 4} \leqslant x\right\}, G(x)$ - функция распределения нормальной $(0, \lambda \times$ $\left.\mathrm{E} \bar{\eta}_{1}^{2} \sqrt{2 \pi} / 2^{3 / 2}\right)$ случайной величины $T=t^{1 / 8} / M$. Тогда в силу предложения 2 сушествуют постоянные $A_{10}$ и $A_{11}$ такие, что

$$
\begin{aligned}
\sup \mid \mathrm{P} & \left\{\frac{V(t)}{t^{3 / 4}} \leqslant x\right\}-G(x) \mid \\
& \leqslant A_{10} \int_{-t^{1 / 8} / M}^{t^{1 / 8} / M}\left|\frac{1}{r}\left(\mathrm{E} e^{i r V(t) / t^{3 / 4}}-\exp \left(-\lambda \mathrm{E} \bar{\eta}_{1}^{2} \sqrt{2 \pi} \frac{r^{2}}{2^{3 / 2} 2}\right)\right)\right| d r+\frac{A_{11}}{t^{1 / 8}} \\
& \leqslant A_{10} A_{9} M^{4} t^{-1 / 2} \int_{\mathbb{R}}|r|^{3} \exp \left(-\lambda \mathrm{E} \bar{\eta}_{1}^{2} \sqrt{2 \pi} \frac{r^{2}}{2^{3 / 2} 2}\right) d r+\frac{A_{11}}{t^{1 / 8}} \leqslant \frac{A_{12}}{t^{1 / 8}} .
\end{aligned}
$$

Пусть $\Phi$ - функция распределения нормальной $(0,1)$ случайной величины. Положим $\lambda \times$ $\mathrm{E} \bar{\eta}_{1}^{2} \sqrt{2 \pi} / 2^{3 / 2}=\sigma^{2}$. Тогда в силу предложения 2

$$
\sup \left|\mathrm{P}\left\{V(t) \leqslant x t^{3 / 4} \sigma\right\}-\Phi(x)\right| \leqslant \frac{A_{12}}{t^{1 / 8}}
$$

Обозначим $B_{t}=t^{3 / 2} \sigma^{2}, \chi_{t}=\sqrt{2 B_{t} \ln \ln B_{t}}$. Так как $B_{t} \rightarrow \infty$ при $t \rightarrow \infty$ из (14) и асимптотической эквивалентности

$$
1-\Phi(x) \sim \frac{1}{x \sqrt{2 \pi}} \exp \left(-\frac{x^{2}}{2}\right) \quad \text { при } x \rightarrow \infty
$$

можно получить, что для любых $b, c>0$ и достаточно больших $t$

$$
\left(\ln B_{t}\right)^{-(1+c) b^{2}} \leqslant \mathrm{P}\left\{V(t) \geqslant b \chi_{t}\right\} \leqslant\left(\ln B_{t}\right)^{-b^{2}} .
$$

Для получения максимального неравенства для $V(t)$ докажем лемму об интегралах по ассоциированной случайной мере. Следуя [12], будем назьвать случайную меру $\mu$ на пространстве $(\mathbb{R}, \mathscr{B})$ ассоциированной, если для любых множеств $B_{1}, \ldots, B_{p}$ из $\mathscr{B}_{b}$ случайные величины $\mu\left(B_{1}\right), \ldots, \mu\left(B_{p}\right)$ образуют ассоциированньй вектор. Напомним, что случайный вектор $\left(\xi_{1}, \ldots, \xi_{p}\right)$ называется ассоииированным, если для любых покоординатно неубывающих действительных функций $f, g: \mathbb{R}^{p} \rightarrow \mathbb{R}$ из конечности ковариации $\operatorname{cov}\left(f\left(\xi_{1}, \ldots, \xi_{p}\right), g\left(\xi_{1}, \ldots, \xi_{p}\right)\right)$ следует ее неотрицательность (см. [13]).

Лемма. Пусть $\mathbb{R}=X_{1} \cup X_{2}, X_{1} \cap X_{2}=\varnothing, X_{1}, X_{2} \in \mathscr{B}$. Пусть мера $\mu$ ассоциирована, и для любъх $B_{1} \subset X_{1} u B_{2} \subset X_{2}\left(B_{1}, B_{2} \in \mathscr{B}_{b}\right) \quad \operatorname{cov}\left(\mu\left(B_{1}\right), \mu\left(B_{2}\right)\right)=0$. Пусть каждая из функиий $f_{i}: \mathbb{R} \rightarrow \mathbb{R}, i=1, \ldots, n$, сохраняет знак на $X_{1} u X_{2}$, причем $\operatorname{sign} f_{i}=\operatorname{sign} f_{j}$ для любых $i, j$. Тогда

$$
\int_{\mathbb{R}} f_{1}(x) \mu(d x), \quad \cdots, \quad \int_{\mathbb{R}} f_{n}(x) \mu(d x)
$$

образуют ассочиированный вектор, если эти интегралы существуют почти наверное. 
ДокАЗАТЕЛЬСТво. Из некоррелированности ассоциированных случайных величин следует их независимость. Значит, для любых $B_{1} \subset X_{1}, B_{2} \subset X_{2}$ случайные величины $\mu\left(B_{1}\right)$ и $\mu\left(B_{2}\right)$ независимы. Пусть

$$
f_{i}^{(m)}(x)=\sum_{k} b_{i, k}^{(m)} \mathbf{1}_{\left\{x \in B_{i, k, m}\right\}}+\sum_{l} c_{i, l}^{(m)} \mathbf{1}_{\left\{x \in C_{i, l, m}\right\}} \rightarrow f_{i}(x)
$$

для каждого $x \in \mathbb{R}$, где все $B_{i, k, m} \in X_{1}$, а все $C_{i, l, m} \in X_{2}, \operatorname{sign} b_{i_{1}, k_{1}}^{\left(m_{1}\right)}=\operatorname{sign} b_{i_{2}, k_{2}}^{\left(m_{2}\right)}$, $\operatorname{sign} c_{i_{1}, l_{1}}^{\left(m_{1}\right)}=\operatorname{sign} c_{i_{2}, l_{2}}^{\left(m_{2}\right)}$ для любых $i_{1}, i_{2}, k_{1}, k_{2}, m_{1}, m_{2}, l_{1}, l_{2}$. Тогда случайные величины

$$
\left\{\left|\int_{X_{1}} f_{i}^{(m)}(x) \mu(d x)\right|\right\}_{i=1}^{n}, \quad\left\{\left|\int_{X_{2}} f_{i}^{(m)}(x) \mu(d x)\right|\right\}_{i=1}^{n}
$$

образуют два ассоциированных вектора, как линейные комбинации ассоциированных величин с положительными коэффициентами. Эти два вектора независимы. Значит, и

$$
\left\{\int_{X_{1}} f_{i}^{(m)}(x) \mu(d x)\right\}_{i=1}^{n}, \quad\left\{\int_{X_{2}} f_{i}^{(m)}(x) \mu(d x)\right\}_{i=1}^{n}
$$

образуют два независимых ассоциированных вектора. Поэтому сумма этих векторов

$$
\left\{\int_{\mathbb{R}} f_{i}^{(m)}(x) \mu(d x)\right\}_{i=1}^{n}
$$

- ассоциированный вектор. Поскольку $f_{i}^{(m)} \rightarrow f_{i}$ поточечно, предельные случайные величины

$$
\left\{\int_{\mathbb{R}} f_{i}(x) \mu(d x)\right\}_{i=1}^{n}
$$

образуют ассоциированньй вектор. Лемма доказана.

Случайная мера $\mu$, описьвающая дробовой шум с нулевым радиусом взаимодействия, удовлетворяет условиям леммы, поскольку для любых непересекающихся борелевских множеств $B_{1}, \ldots, B_{m} \in \mathscr{B}_{b}$ случайные величины $\mu\left(B_{1}\right), \ldots, \mu\left(B_{m}\right)$ независимы.

Для любой положительной строго возрастающей к бесконечности последовательности $\left(t_{n}\right)_{n=1}^{\infty}$ введем функции

$$
f_{1}(x)=x e^{-x^{2} /\left(2 t_{1}\right)}, \quad f_{k}(x)=x\left(e^{-x^{2} /\left(2 t_{k}\right)}-e^{-x^{2} /\left(2 t_{k-1}\right)}\right), \quad k=2, \ldots, n .
$$

Так как для $0<s<t e^{-x^{2} /(2 t)}>e^{-x^{2} /(2 s)}$, для любого $x \in \mathbb{R}$ и любого $k \in\{1, \ldots, n\}$ выполнено $\operatorname{sign} f_{k}(x)=\operatorname{sign} x$.

Значит, по лемме случайные величины $\Delta V_{k}=\int_{\mathbb{R}} f_{k}(x) \mu(d x)$ образуют ассоциированньй вектор. Заметим теперь, что $\sum_{k=1}^{n} \Delta V_{k}=V\left(t_{n}\right)$, т.е. $V\left(t_{n}\right)$ - последовательность сумм ассоциированных случайных величин. Для нее вьполнено максимальное неравенство (см. [14]): если $0<\lambda_{1}<\lambda_{2}$ и $\left(\lambda_{2}-\lambda_{1}\right)^{2} \geqslant \mathrm{E} V^{2}\left(t_{n}\right)=B_{t_{n}}$, то

$$
\mathrm{P}\left\{\max \left(\left|V\left(t_{1}\right)\right|, \ldots,\left|V\left(t_{n}\right)\right|\right) \geqslant \lambda_{2}\right\} \leqslant\left(1-\frac{B_{t_{n}}}{\left(\lambda_{2}-\lambda_{1}\right)^{2}}\right)^{-1} \mathrm{P}\left\{V\left(t_{n}\right) \geqslant \lambda_{1}\right\} .
$$


Положим $\bar{V}(s)=\max _{0<t \leqslant s} V(t), \bar{V}_{k}(s)=\max _{l=1, \ldots, 2^{k}} V\left(l s / 2^{k}\right)$. Тогда

$$
\left\{\bar{V}(s)>(1+\gamma) \chi_{s}\right\} \subset \bigcup_{k=0}^{\infty}\left\{\bar{V}_{k}(s)>(1+\gamma) \chi_{s}\right\}
$$

в силу непрерьвности траекторий $V(t)$. Кроме того,

$$
\left\{\bar{V}_{k}(s)>(1+\gamma) \chi_{s}\right\} \subset\left\{\bar{V}_{k+1}(s)>(1+\gamma) \chi_{s}\right\}
$$

Так как ввиду (17) для любого $k$

$$
\mathrm{P}\left\{\bar{V}_{k+1}(s)>(1+\gamma) \chi_{s}\right\} \leqslant\left(1-\frac{B_{s}}{(\gamma / 2)^{2} 2 B_{s} \ln \ln B_{s}}\right)^{-1} \mathrm{P}\left\{V(s)>\left(1+\frac{\gamma}{2}\right) \chi_{s}\right\},
$$

то и

$$
\mathrm{P}\left\{\bar{V}(s)>(1+\gamma) \chi_{s}\right\} \leqslant A_{13} \mathrm{P}\left\{V(s)>\left(1+\frac{\gamma}{2}\right) \chi_{s}\right\} .
$$

Возьмем $a>0$. Из (16) следует, что

$$
\mathrm{P}\left\{V\left(a^{k}\right)>\left(1+\frac{\gamma}{2}\right) \chi_{a^{k}}\right\} \leqslant\left(A_{14}(a, \sigma) \frac{3}{2} k\right)^{-(1+\gamma / 2)^{2}}
$$

Так как процесс $V$ непрерьвен почти наверное, события, входящие в следующие неравенства, измеримы:

$$
\begin{aligned}
\mathrm{P}\left(\bigcap_{t>0} \bigcup_{s>t}\{V(s)>(1+\varepsilon) \chi(s)\}\right) & \leqslant \mathrm{P}\left(\max _{s \in\left(a^{k-1}, a^{k}\right]} V(s)>(1+\varepsilon) \chi\left(a^{k-1}\right) \text { б.ч. }\right) \\
& \leqslant \mathrm{P}\left(\bar{V}\left(a^{k}\right)>(1+\varepsilon) \chi\left(a^{k-1}\right) \text { б.ч. }\right),
\end{aligned}
$$

где запись “ $E_{k}$ б.ч." означает, что произошло бесконечное число событий $E_{k}$.

Так как $\chi\left(a^{k}\right) / \chi\left(a^{k-1}\right)<a$ для достаточно больших $k$, то

$$
\begin{aligned}
\mathrm{P}\left(\bigcap_{t>0} \bigcup_{s>t}\{V(s)>(1+\varepsilon) \chi(s)\}\right) & \leqslant \mathrm{P}\left(\bar{V}\left(a^{k}\right)>\frac{1+\varepsilon}{a} \chi\left(a^{k}\right) \text { б.ч. }\right) \\
& \leqslant \mathrm{P}\left(\bar{V}\left(a^{k}\right)>\left(1+\frac{\varepsilon}{2}\right) \chi\left(a^{k}\right) \text { б.ч. }\right),
\end{aligned}
$$

если $a-1$ достаточно мало. В силу (18), (19) и леммы Бореля-Кантелли отсюда следует, что для любого $\varepsilon>0$

$$
\mathrm{P}\left(\bigcap_{t>0} \bigcup_{s>t}\{V(s)>(1+\varepsilon) \chi(s)\}\right)=0 .
$$

Аналогичное соотношение с заменой $V(s)$ на $-V(s)$ имеет место в силу симметричности распределения процесса $V$. Поэтому

$$
\varlimsup_{t \rightarrow \infty} \frac{|V(t)|}{\sqrt{2 B_{t} \ln \ln B_{t}}} \leqslant 1 \text { почти наверное. }
$$


Для любого $a>1$ введем случайные величины

$$
S_{a}(k)=\int_{\left(-a^{k+1},-a^{k}\right] \cup\left(a^{k}, a^{k+1}\right]} x \exp \left(-\frac{x^{2}}{2 a^{k+1}}\right) \mu(d x)
$$

и $R_{a}(k)=V\left(a^{k}\right)-S_{a}(k)$. Применяя тот же способ, с помощюю которого мы вычислили семиинварианты $V(t)$, получаем

$$
\mathrm{D} R_{a}(k)=\lambda \mathrm{E} \bar{\eta}_{1}^{2} \int_{\mathbb{R} \backslash\left(\left(-a^{k+1},-a^{k}\right] \cup\left(a^{k}, a^{k+1}\right]\right)} x^{2} \exp \left(-\frac{x^{2}}{a^{2 k+1}}\right) d x=\sigma_{a}^{2} a^{3(2 k+1) / 2},
$$

$\sigma_{a}^{2}>0$, причем $\lim _{a \rightarrow \infty} \sigma_{a}=0$.

Нечетные семиинварианты $R_{a}(k)$ равны 0 , а четные равны

$$
\begin{aligned}
\gamma_{2 n}\left(R_{a}(k)\right) & =\lambda \mathrm{E} \bar{\eta}_{1}^{2 n} \int_{\mathbb{R} \backslash\left(\left(-a^{k+1},-a^{k}\right] \cup\left(a^{k}, a^{k+1}\right]\right)} x^{2 n} \exp \left(-\frac{n x^{2}}{a^{2 k+1}}\right) d x \\
& \leqslant \int_{\mathbb{R}} x^{2 n} \exp \left(-\frac{n x^{2}}{a^{2 k+1}}\right) d x=\gamma_{2 n}\left(V\left(a^{2 k+1}\right)\right) .
\end{aligned}
$$

Поэтому, повторяя вькладки (10)-(13), получаем

$$
\left|\mathrm{E} \exp \left(\frac{i r R_{a}(k)}{a^{3(2 k+1) / 4}}\right)-\exp \left(-\sigma_{a}^{2} \frac{r^{2}}{2}\right)\right| \leqslant A_{15} a^{-(2 k+1) / 2}(r M)^{4} \exp \left(-\sigma_{a}^{2} \frac{r^{2}}{2}\right) .
$$

Используя предложение 2 , находим, что

$$
\sup \left|\mathrm{P}\left\{R_{a}(k) \leqslant x a^{3(2 k+1) / 4} \sigma_{a}\right\}-\Phi(x)\right| \leqslant \frac{A_{16}}{a^{(2 k+1) / 8}} .
$$

Из оценки (15) для любого $b>0$ и $k>k_{0}(a)$ следует, что

$$
\mathrm{P}\left\{R_{a}(k)>b \sqrt{2 \mathrm{D} R_{a}(k) \ln \ln \mathrm{D} R_{a}(k)}\right\} \leqslant\left(\ln \mathrm{D} R_{a}(k)\right)^{-b^{2}}=\left(\frac{3}{2}(2 k+1) \ln \sigma_{a}^{2}\right)^{-b^{2}} .
$$

То же верно при замене $R_{a}(k)$ на $-R_{a}(k)$ в силу симметрии распределения $R_{a}(k)$. Поэтому для $b>1$

$$
\sum_{k=1}^{\infty} \mathrm{P}\left\{\left|R_{a}(k)\right|>b \sqrt{2 \mathrm{D} R_{a}(k) \ln \ln \mathrm{D} R_{a}(k)}\right\}<\infty
$$

и по лемме Бореля-Кантелли

$$
\mathrm{P}\left(\left|R_{a}(k)\right|>b \sqrt{2 \mathrm{D} R_{a}(k) \ln \ln \mathrm{D} R_{a}(k)} \text { б.ч. }\right)=0 .
$$

Для любых $\tau, \mu$ таких, что $0<\tau<\mu<1$, имеем

$$
\begin{aligned}
& \mathrm{P}\left\{V\left(a^{2 k+1}\right)>(1-\tau) \sqrt{2 B_{a^{2 k+1}} \ln \ln B_{a^{2 k+1}}}\right\} \\
& \quad \leqslant \mathrm{P}\left\{S_{k}(a)>(1-\mu) \sqrt{2 B_{a^{2 k+1}} \ln \ln B_{a^{2 k+1}}}\right\} \\
& \quad+\mathrm{P}\left\{R_{a}(k)(a)>(\mu-\tau) \sqrt{2 B_{a^{2 k+1}} \ln \ln B_{a^{2 k+1}}}\right\} .
\end{aligned}
$$


Из $(21)$ следует, что если $(\mu-\tau) \sqrt{B_{a^{2 k+1}} / \mathrm{D} R_{a}(k)}=(\mu-\tau) \sigma / \sigma_{a}>1$, то

$$
\sum_{k=1}^{\infty} \mathrm{P}\left\{\left|R_{a}(k)\right|>(\mu-\tau) \sqrt{2 B_{a^{2 k+1}} \ln \ln B_{a^{2 k+1}}}\right\}<\infty,
$$

а из (16) следует, что для достаточно малого $c>0$

$$
\begin{gathered}
\sum_{k=1}^{\infty} \mathrm{P}\left\{V\left(a^{2 k+1}\right)>(1-\tau) \sqrt{2 B_{a^{2 k+1}} \ln \ln B_{a^{2 k+1}}}\right\} \\
\geqslant \sum_{k=1}^{\infty}\left(A_{14}(a, \sigma) \frac{3}{2} k\right)^{-(1+c)(1-\tau)^{2}}=\infty .
\end{gathered}
$$

Значит, из (22) имеем

$$
\sum_{k=1}^{\infty} \mathrm{P}\left\{S_{k}(a)>(1-\mu) \sqrt{2 B_{a^{2 k+1}} \ln \ln B_{a^{2 k+1}}}\right\}=\infty .
$$

А так как $S_{k}(a)$ независимы между собой согласно предложению 1 , из леммы БореляКантелли следует, что

$$
\mathrm{P}\left(S_{k}(a)>(1-\mu) \sqrt{2 B_{a^{2 k+1}} \ln \ln B_{a^{2 k+1}}} \text { б.ч. }\right)=1 .
$$

Далее, для $\varepsilon>0$

$$
\begin{aligned}
& \left\{\left(V\left(a^{2 k+1}\right)>(1-\varepsilon) \sqrt{2 B_{a^{2 k+1}} \ln \ln B_{a^{2 k+1}}} \text { б.ч. }\right)\right\} \\
& \supset\left\{\left(S_{k}(a)>\left(1-\frac{\varepsilon}{2}\right) \sqrt{2 B_{a^{2 k+1}} \ln \ln B_{a^{2 k+1}}} \text { б.ч. }\right)\right\} \\
& \cap\left\{\left(\left|R_{a}(k)\right|>\frac{\varepsilon}{2} \sqrt{2 B_{a^{2 k+1}} \ln \ln B_{a^{2 k+1}}} \text { к.ч. }\right)\right\},
\end{aligned}
$$

где запись “ $E_{k}$ к.ч." означает, что произошло конечное число событий из $E_{k}$. Выберем в (22) $\tau=\varepsilon / 6, \mu=\varepsilon / 2$, а число $a$ таким, что $\sigma_{a} / \sigma<\varepsilon / 6$. Тогда вероятность каждого из пересекаемых событий в (23) равна 1 и, следовательно, для любого $\varepsilon>0$

$$
\mathrm{P}\left\{\left(V\left(a^{2 k+1}\right)>(1-\varepsilon) \sqrt{2 B_{a^{2 k+1}} \ln \ln B_{a^{2 k+1}}} \text { б.ч. }\right)\right\}=1,
$$

т.е.

$$
\varlimsup_{t \rightarrow \infty} \frac{|V(t)|}{\sqrt{2 B_{t} \ln \ln B_{t}}} \geqslant 1 \text { почти наверное. }
$$

Таким образом доказан закон повторного логарифма для числителя (3). Для знаменателя (3) подходят те же самые рассуждения, которые приводят к тому, что если

$$
S(t)=\int_{\mathbb{R}} e^{-x^{2} /(2 t)} \mu(d x),
$$

то

$$
\varlimsup_{t \rightarrow \infty} \frac{\left|S(t)-\lambda \mathrm{E} \eta_{1} \sqrt{2 \pi t}\right|}{\sqrt{2 D \sqrt{t} \ln \ln t}} \leqslant 1 \text { почти наверное }
$$


где $D=\lambda \mathrm{E} \eta_{1} \sqrt{2 \pi t}$. Отсюда следует, что

$$
\frac{S(t)}{\lambda \mathrm{E} \eta_{1} \sqrt{2 \pi t}} \rightarrow 1 \text { почти наверное. }
$$

Значит, учитьвая (3), (20), (24) и (25), получаем

$$
\varlimsup_{t \rightarrow \infty} \frac{|v(t, 0)|}{\sqrt{2 B_{t} \ln \ln B_{t}} /\left(t \lambda \mathrm{E} \eta_{1} \sqrt{2 \pi t}\right)}=1 \text { почти наверное. }
$$

Теорема доказана.

Автор благодарен профессору А. В. Булинскому за постановку задачи и внимание к работе.

\section{СПИСОК ЦИТИРОВАННОЙ ЛИТЕРАТУРЫ}

[1] Гурбатов С. Н., Малахов А. Н., Саичев А. И. Нелинейные случайные волны в средах без дисперсии. М.: Наука, 1990.

[2] Булинский А.В., Молчанов С. А. Асимптотическая гауссовость решения уравнения Бюргерса со случайными начальными данными // Теория вероятн. и ее применения. 1991. T. 36. № 2. C. 217-235.

[3] Bulinski A. V. Some problems of asymptotical analyses of nonlinear diffusion // Probab. Theory Math. Statist / ed. A. N. Shiryaev et al. Proc. 6th USSR-Japan Symp. Singapore: World Sci. Publ., 1993. P. 32-46.

[4] Bulinski A. V. CLT for the solution of the multidimensional Burgers equation with random data // Ann. Acad. Sci. Fenn. Ser. A. I. Math. 1992. V. 17. № 1. P. 11-22.

[5] Albeverio S., Molchanov S. A., Surgailis D. Stratified structure of the Universe and Burgers' equation - a probabilistic approach // Probab. Theory Related Fields. 1994. V. 100. P. 457-484.

[6] Гринив О.О.Центральная предельная теорема для уравнения Бюргерса // ТМФ. 1991. T. 88. №1. C. 7-13.

[7] Leonenko N., Orsingher E. Limit theorems for solutions of the Burgers equation with Gaussian and non-Gaussian initial conditions // Теория вероятн. и ее применения. 1995. Т. 40. № 2. C. 387-403.

[8] Surgailis D., Woyczynsky W. A. Burgers' equation with non-local shot-noise data // J. Appl. Probab. 1994. V. 31. P. 351-362.

[9] Hsing T.L., Teugels J. L. Extremal properties of shot noise processes // Adv. in Appl. Probab. 1989. V. 21. P. 513-525.

[10] Ширяев А. Н. Вероятность. М.: Наука, 1980.

[11] Петров В. В. Предельные теоремы для сумм независимых случайных величин. М.: Наука, 1987.

[12] Burton R., Waymire E. Scaling limits for associated random measures // Ann. Probab. 1985. V. 13. № 4. P. 1267-1278.

[13] Esary J. D., Proschan F., Walkup D. W. Association of random variables, with applications // Ann. Math. Statist. 1967. V. 38. P. 1466-1474.

[14] Newman C. M., Wright A. L. An invariance principle for certain dependent sequences // Ann. Probab. 1981. V. 9. №4. P. 671-675. 